\title{
Oral Leukoplakia as It Relates to HPV Infection: A Review
}

\author{
L. Feller and J. Lemmer \\ Department of Periodontology and Oral Medicine, University of Limpopo, Medunsa Campus, Medunsa, South Africa
}

Correspondence should be addressed to L. Feller, lfeller@ul.ac.za

Received 13 July 2011; Revised 2 September 2011; Accepted 19 December 2011

Academic Editor: Neil S. Norton

Copyright ( $\odot 2012$ L. Feller and J. Lemmer. This is an open access article distributed under the Creative Commons Attribution License, which permits unrestricted use, distribution, and reproduction in any medium, provided the original work is properly cited.

\begin{abstract}
Leukoplakia is the most common potentially malignant lesion of the oral cavity and can be categorised according to its clinical appearance as homogeneous or nonhomogenous. Tobacco and areca nut use, either alone or in combination are the most common risk factors for oral leukoplakia, but some oral leukoplakias are idiopathic. Some leukoplakias arise within fields of precancerized oral epithelium in which the keratinocytes may be at different stages of cytogenetic transformation. Leukoplakias may unpredictably regress, may remain stable, or may progress to carcinoma. There is a greater risk of carcinomatous transformation of idiopathic leukoplakia, of non-homogenous leukoplakia, of leukoplakia affecting the floor of the mouth; the ventrolateral surface of the tongue and the maxillary retromolar and adjoining soft palate (collectively called high-risk sites), of leukoplakia with high-grade epithelial dysplasia, and of leukoplakia in which the keratinocytes carry cytogenetic alterations associated with carcinomatous transformation. Although there appears to be some link between human papillomavirus (HPV) and oral leukoplakia, there is little evidence to support a causal relationship either between HPV infection and oral leukoplakia or between HPV-infected leukoplakic keratinocytes and their carcinomatous transformation.
\end{abstract}

\section{Introduction}

Leukoplakia is the most common potentially malignant lesion of the oral cavity $[1,2]$. Leukoplakia is a term describing "a white lesion of the oral mucosa that cannot be characterized clinically or microscopically as any other defined oral disease entity" $[3,4]$. At a World Health Organisation (WHO) workshop held in 2005, it was recommended that oral leukoplakia be defined as "a white plaque of questionable risk having excluded (other) known diseases or disorders that carry no increased risk for cancer" [5-7]. Oral leukoplakia needs to be distinguished from other predominantly white keratotic lesions including frictional keratosis and stomatitis nicotina, which do not have malignant potential $[1,2,5-8]$.

About $70-90 \%$ of oral leukoplakias are related to smoking and areca nut use, either alone or in combination, and there is a direct relationship between the frequency and the duration of cigarette, pipe, or cigar smoking and the prevalence of oral leukoplakia $[8,9]$. The factors implicated in the pathogenesis of idiopathic leukoplakia are unknown.

However it is possible that infection of the oral epithelium with human papillomavirus (HPV) and excessive consumption of alcoholic beverages may be associated with oral leukoplakia, but there is little evidence of a causal relationship between either HPV infection or alcohol, and oral leukoplakia [6].

It has been suggested that a definitive diagnosis of oral leukoplakia must be established by histopathological exclusion of other keratotic oral lesions that are recognised as specific entities, and by exclusion of any aetiological agents other than tobacco/areca nut use [7].

It is the opinion of the authors that these criteria are unrealistically limiting, ignoring as they do the possible roles of HPV, alcohol, chronic inflammation, and lowgrade chronic frictional trauma in the pathogenesis of oral leukoplakia. Referring to the WHO definition of 2005 above, it is difficult to understand how such a definition could gain any useful currency since it is so "exclusive" that it leaves no rational guidance for the everyday diagnosis of oral leukoplakia.

\section{Oral Leukoplakia: Clinical Aspects}

According to its clinical appearance, oral leukoplakia can be categorised into two main clinical types: homogeneous 
and non-homogeneous. Either type may occur as an isolated lesion or as multiple lesions. The leukoplakic lesion can vary in size from a few millimetres to several centimeters [1, 2, 512].

Homogeneous leukoplakia is a uniformly white flat plaque with a smooth or relatively smooth surface; nonhomogeneous leukoplakia may be nodular or verrucous having a wrinkled or corrugated surface or may be a mingling of white and red areas termed erythroleukoplakia $[7,10,11]$.

The clinical appearance of oral leukoplakia may change over time. Some homogeneous lesions may become larger, or non-homogeneous, but most oral leukoplakias will remain stable or will regress, while some few will undergo carcinomatous transformation [13-15].

Oral erythroplakia, of all precancerous oral lesions, carries the greatest threat of malignant transformation. It has a velvety-red appearance, and about $50 \%$ of all cases of erythroplakia are already squamous cell carcinomata at the time of diagnosis. The erythroplakic component of oral erythroleukoplakia is identical to erythroplakia [16].

Proliferative verrucous leukoplakia, considered to be either a clinical subtype of non-homogeneous oral leukoplakia or to be a distinct clinical entity, is not strongly associated with smoking, is characterized by multiple leukoplakic lesions that affect wide areas of the oral epithelium, and may progress either to verrucous carcinoma or to squamous cell carcinoma [17-20]. In most cases, proliferative verrucous leukoplakia is recognised only late in its course since in its initial stages it is identical to an isolated leukoplakia [17-24].

\section{Epidemiology of Oral Leukoplakia}

The data from epidemiological studies on oral leukoplakia is inconsistent, most probably owing to differences in case selection criteria (house-to-house surveys, hospital surveys, age, gender, race, ethnicity, and tobacco use) and in methodology (diagnostic criteria, time of follow-up and whether or not the leukoplakia had previously been treated) $[1,10,11]$.

Estimates of the global prevalence of oral leukoplakia range from $0.5 \%$ to $3.46 \%$, and of the rates of carcinomatous transformation of oral leukoplakia from $0.7 \%$ to $2.9 \%$ [25]. Oral leukoplakia is more prevalent in India where persons smoke and practice the habit of tobacco and areca nut chewing more than elsewhere [26].

Oral leukoplakia is usually diagnosed in middle age, and its prevalence increases with age. About $10 \%$ of oral leukoplakias are idiopathic and the greater part of the remaining $90 \%$ is associated with the use of tobacco/areca nut [26]. Males are more often affected than females probably owing to the greater prevalence of tobacco use by males [8]. The buccal mucosa is affected in $25 \%$ of cases, the mandibular gingiva in $20 \%$, the tongue in $10 \%$, the floor of the mouth in $10 \%$, and other oral sites account for the remainder [13].

The literature on the relationship between race and oral leukoplakia is sparse. In a South African study of archived histopathological material, $86 \%$ of oral leukoplakias were from whites, 9\% from blacks, and 5\% from Asians, despite the fact that the vast majority of South Africans are black
[27]. This is not easy to explain. Bearing in mind that the study was on histopathological material, an explanation may be that blacks tend to postpone seeking medical treatment until the leukoplakia has already undergone carcinomatous transformation, thus skewing the statistics away from the leukoplakia [27], or because black people in South Africa may smoke less than white people.

\section{Epithelial Dysplasia and Oral Leukoplakia}

The reported prevalence of epithelial dysplasia in oral leukoplakia ranges from 5\% to $25 \%$ [8]. Dysplasia is more frequent in non-homogeneous than in homogeneous leukoplakia [11], and it is probable that dysplasia is the histopathological expression of genomic and molecular alterations in a field of keratinocytes $[28,29]$.

The presence of epithelial dysplasia is a marker of the malignant potential of oral leukoplakia, and the risk of an individual leukoplakic lesion to progress to carcinoma increases with the increase of the grade of the epithelial dysplasia $[11,12,14,30]$.

However, some dysplastic oral leukoplakias can remain stable or even regress, while some oral leukoplakias without epithelial dysplasia will indeed progress to carcinoma [5, $11,12]$. In one study $36 \%$ of dysplastic oral leukoplakias progressed to squamous cell carcinoma, but a substantial proportion of $16 \%$ of oral leukoplakias without epithelial dysplasia at the time of initial biopsy also progressed to carcinoma [31]. The risk of progression to carcinoma of leukoplakias with moderate and severe dysplasia is estimated to be twice as great as for oral leukoplakias with simple epithelial hyperplasia or with mild dysplasia [14].

Treatment of dysplastic oral leukoplakia by excision, by laser or by cryosurgery, or by topical or systemic chemotherapy does not eliminate either the risk of relapse or recurrence, or the risk of carcinomatous transformation $[1,5,6]$. The estimated recurrence rate of oral leukoplakia may be as high as 30\% [32], and squamous cell carcinoma develops at $12 \%$ of sites of treated leukoplakia [15]. In a study investigating the pattern of carcinomatous transformation of oral leukoplakia and oral erythroplakia, 36\% of carcinomata developed at the same site, $49 \%$ at contiguous sites, and $15 \%$ at oral sites remote from the preexisting lesions [33].

It is evident from this data that some cases of treated oral leukoplakia are unpredictably destined to recur or to undergo carcinomatous transformation, and that there are not yet any diagnostic methods available (clinical, histological or molecular), to confidently identify these cases [1].

Epithelial dysplasia in oral leukoplakia is a useful marker of the risk of carcinomatous transformation and is an important guide to clinical management $[1,11,28]$. However, since dysplasia can remain stable for long periods, it cannot be used with confidence as a predictor of carcinomatous transformation $[14,29]$. Moreover, as the histological exercise of grading of epithelial dysplasia is highly subjective with low interpersonal and intrapersonal reproducibility [16, 34, 35], and as an incisional biopsy cannot be representative of an 
entire lesion [34, 36], a histopathological report of any degree of epithelial dysplasia or of the absence of epithelial dysplasia must be viewed with caution.

\section{The Natural Course and the Malignant Potential of Oral Leukoplakia}

The progression of oral leukoplakia to carcinoma is unpredictable but is relatively infrequent with an estimated overall risk of less than $2 \%$ per year $[5,6,13,26]$; if progression occurs, it may take a few months or many years [8]. The carcinomatous transformation of oral leukoplakia is not predictably associated with tobacco smoking [33], and the frequency of carcinomatous transformation of idiopathic leukoplakia is higher than that of tobacco-associated leukoplakia $[11,26]$.

In populations where smoking, the use of smokeless tobacco, reverse smoking, and the use of areca nut are very prevalent, most squamous cell carcinomata arise from preexisting leukoplakias; while in populations with a lower prevalence of these habits, most squamous cell carcinomata arise de novo in normal-looking epithelium [26]. It has been suggested that squamous cell carcinoma arising de novo usually runs a more aggressive course and has a less favourable prognosis than squamous cell carcinoma arising from preexisting leukoplakia $[12,26]$, but a recent study has demonstrated little difference [37]. Sometimes squamous cell carcinoma arises de novo in close proximity to oral leukoplakias [8].

Non-homogeneous leukoplakia has a greater risk of carcinomatous transformation (20-25\%) than homogeneous leukoplakia $(0.6-5 \%)[11,13]$. Most leukoplakias either remain stable or will regress $[13,15]$. However, if proliferative verrucous leukoplakia is considered as a distinct entity, most such cases progress to carcinoma $[18,24]$.

The rates of progression of large oral leukoplakias $(>5 \mathrm{~mm})$ and of leukoplakias at sites in the mouth known to be at most risk of developing carcinoma (floor of mouth, ventrolateral surface of tongue, and maxillary retromolar/soft palate region) are greater than for smaller leukoplakias or for leukoplakias at other sites in the mouth $[1,11,13,33,38]$. The increased risk of carcinomatous transformation of oral leukoplakia at high-risk sites is not entirely a function of the degree of dysplasia. It is also dependent upon as yet undefined characteristics of the location of the leukoplakia since the rate of carcinomatous transformation of dysplastic leukoplakias at high-risk sites is greater than the rate of transformation of equally dysplastic leukoplakias at other sites [38].

There is evidence that clearly suggests that some leukoplakias arise from cytogenetically altered transformed keratinocytes within fields of precancerized oral epithelium. Keratinocytes of oral leukoplakia show cytogenetic changes including alterations in the p53 tumour suppressor gene, aberrations in their DNA content, and loss of heterozygosity $(\mathrm{LoH})$ at chromosomal regions of candidate tumour suppressor genes $[20,33,39-45]$. $\mathrm{LoH}$ at either $3 p$ or at $9 p$ occurs frequently in keratinocytes of oral leukoplakia and is associated with carcinomatous transformation of these lesions [33, 41, 42, 44, 45]. Additional cytogenetic alterations to the keratinocytes in the precancerized field referred to above may result in the evolution of one or several keratinocytes containing a complete set of cytogenetic alterations of a cancerous phenotype, and in the subsequent development of squamous cell carcinoma [41, 46, 47].

However, some precancerous oral leukoplakias in which cytogenetic alterations in the keratinocytes cannot be demonstrated, nevertheless undergo carcinomatous transformation $[41,42]$. The pathogenic mechanisms that bring about the progressive transformation of these keratinocytes to carcinomatous cells are yet to be elucidated. Most oral leukoplakias are benign in nature and will remain stable or will regress $[28,32]$. These leukoplakias probably have a different aetiopathogenesis to precancerous leukoplakias and probably do not have the cytogenetic characteristics of precancerous leukoplakias. What is certain, however, is that leukoplakias with malignant potential and those without malignant potential cannot be distinguished clinically [1].

\section{Human Papillomavirus and Oral Leukoplakia}

Human papilloma viruses (HPVs) are strictly epitheliotropic and infect either cutaneous or mucosal squamous epithelium, depending upon their genotype [48, 49]. Those that infect mucosal epithelium have been categorized into highrisk types (e.g., HPV-16, 18, 31, 33, and 35) based on their epidemiological association with carcinoma of the cervix uteri, or into low-risk types (HPV-6, 11, 13, and 32) [50]. These categories have been universally adopted for use in studies of the oncogenic significance of HPV infection at all anatomical regions of the upper aerodigestive tract.

Low-risk HPV genotypes have been implicated in the pathogenesis of the benign oral proliferative epithelial lesions, squamous cell papilloma, common wart (verrucous vulgaris), condyloma acuminatum, and focal epithelial hyperplasia (Heck disease); while high-risk types have been associated with precancerous and cancerous oral and oropharyngeal epithelial lesions [49, 51-56].

There is an extreme variation in the reported prevalence of HPV infection in oral precancerous and cancerous lesions ranging from $0 \%$ to $100 \%[57,58]$. This is owing to differences in sampling and HPV detection methods, to differences in ethnicity, geographic locations, and sample size of the subjects examined, and to the inappropriate grouping together of different lesions from different anatomical locations of the mucosa of the upper aerodigestive tract $[49,51,58-64]$.

Many studies investigating the association of HPV and squamous cell carcinoma of the mucosa of the upper aerodigestive tract used PCR techniques for detection of HPV DNA without also quantifying the DNA viral load. PCR can detect extremely small fragments of DNA that may represent either contamination of the sample or biologically insignificant HPV infection $[51,57,58]$. These findings have been reported as if they were pathogenically significant. 
Whether these are legitimate findings or are the results of inconsistencies and errors in methodology, several HPV genotypes have been detected in precancerous oral lesions. High-risk HPV genotypes, in particular HPV-16, have been reported to be the most prevalent in oral leukoplakias, including proliferative verrucous leukoplakia [55, 65]. Other reports implicated low-risk rather than high-risk HPV genotypes in oral leukoplakia [54, 63], and yet others assert that oral leukoplakia is coinfected with a variety of HPV genotypes $[49,55,66]$. In a meta-analysis of data from 94 studies of a total of 4580 specimens, Miller and Johnstone [63] determined that the likelihood of HPV being detected in precancerous oral lesions is 2 to 3 times greater and in oral squamous cell carcinoma is 4 to 5 times greater than in normal oral mucosa. The prevalence of HPV in normal oral mucosa, in nondysplastic leukoplakias, in dysplastic leukoplakias and in other precancerous intraepithelial oral neoplasms, and in oral squamous cell carcinoma is likely to be $10 \%, 20.2 \%, 26.2 \%$, and $46.5 \%$, respectively [63].

This suggests that there may be some link between HPV infection and oral precancerous and cancerous lesions. As E6 and E7 oncoproteins of high-risk HPV genotypes have the capacity to mediate carcinomatous transformation of infected keratinocytes by inactivating cellular p53 and Rb tumour suppressor pathways [50, 52], HPV may play either an oncogenic or a co-oncogenic role in some HPV-infected precancerous and cancerous epithelial neoplasms.

In fact, HPV-16 has been found to be causally associated primarily with squamous cell carcinoma of the palatal tonsils [67-70] in a subset of subjects who are younger, consume less tobacco, are more engaged in high-risk sexual behaviour (great number of lifetime sexual partners and practicing oral-genital sex), have higher HPV-16 serum antibody titers, and have a better disease-free survival and overall survival rates than subjects with $\mathrm{HPV}$-cytonegative oropharyngeal squamous cell carcinoma [51, 64, 67-70]. The cells of HPVcytopositive oropharyngeal carcinoma in these subjects have a distinct molecular profile [50]. The cells of squamous cell carcinoma causally associated with HPV express E6/E7 oncoproteins. They frequently demonstrate viral integration within the cellular genome with the presence of intact E6 gene. They exhibit high viral load, reduced expression of $\mathrm{Rb}$ proteins, functional overexpression of p16 INK4A, unmutated p53 gene, and loss of heterozygosity ( $\mathrm{LoH})$ at chromosomal loci $3 \mathrm{p}, 9 \mathrm{p}$ and $17 \mathrm{p}$ is infrequent [51, 6874]. In contrast, HPV-cytonegative oropharyngeal squamous cell carcinoma is characterized by p53 gene mutations, by frequent $\mathrm{LoH}$ at $3 \mathrm{p}, 9 \mathrm{p}$, and $17 \mathrm{p}$, by decreased levels of $\mathrm{p} 16 \mathrm{INK} 4 \mathrm{~A}$ and by normal or increased levels of $\mathrm{Rb}$ proteins $[50,51,71]$.

Recent meta-analyses and comprehensive studies [60, 62, 67,75 ] show little or no causal association between HPV and oral squamous cell carcinoma in contrast to the strong association between HPV and oropharyngeal squamous cell carcinoma. HPV-16 cytopositive oral squamous cell carcinoma is characterized by a low viral load, by infrequent viral integration, and the cancerous cells seldom contain active transcriptional E6/E7 mRNA $[72,76]$. However, it is possible that, in HPV-cytopositive oral squamous cell carcinomata that do not express E6/E7 mRNA, E6/E7 oncoproteins may well have participated or have had a complementary role in the initial transformation, but then phased out [77].

With oropharyngeal carcinoma as the model, a causal association between HPV and cancerization in oral epithelium is likely if the cells of the lesion contain HPV DNA expressing E6 and/or E7 mRNA [71], if there is viral integration within the cellular genome [74], and if there is a high viral load ( $>1$ copies per cell). A limited biological significance of the virus in the process of transformation can be deduced if there is a low-copy number $(<1$ copy per cell), or if there is no transcriptional activity of E6 and/or E7 mRNA [60, 71]. Nevertheless, although integration of HPV DNA into the cellular genome is a strong indication of the oncogenic potential of the virus, transcription of HPV16 E6/E7 mRNA has been shown to occur in oropharyngeal carcinoma without integration of viral DNA, the virus being in an episomal form [69].

It is well established that non-homogeneous leukoplakias more frequently undergo malignant transformation than homogeneous leukoplakias, yet it appears that HPV is found more commonly in homogeneous than in nonhomogeneous leukoplakia $[54,78]$. Nevertheless the role of HPV in the pathogenesis of oral leukoplakia and in its progression to carcinoma is unclear since there is a low viral load in HPV-cytopositive precancerous and cancerous oral lesions, and viral integration is seldom found $[72,76]$. It is possible that HPV DNA in oral leukoplakia and in oral squamous cell carcinoma may be oncogenically insignificant. Alternatively the HPV may have superinfected keratinocytes already initially transformed and may thus additively or synergistically promote later stages of transformation $[49,51$, 69].

Little is known about the E6 and E7 proteins of lowrisk HPV either with regard to their role in the pathogenesis of HPV-infected oral leukoplakias, or with regard to their role in the carcinomatous transformation of some leukoplakias. It is possible that as in other HPV-associated benign proliferative oral epithelial lesions, E6 and E7 proteins of low-risk HPV types found in oral leukoplakia may stimulate suprabasal postmitotic infected keratinocytes to reenter the S-phase of the cell cycle resulting in epithelial proliferation and disturbed maturation, without causing the genomic instability possibly associated with subsequent cell transformation. This mechanism may be a co-determinant of the development of the leukoplakia, but there is no concrete evidence to support this.

\section{Treatment}

As oral leukoplakia is potentially malignant, and as some leukoplakias will unpredictably progress to carcinoma, ideally all oral leukoplakias should be treated. When dealing with two or three accessible circumscribed lesions, the treatment of choice is surgical excision. For multiple or for large leukoplakias where surgical treatment would be impractical because it would result in unacceptable deformities or in functional disabilities, treatment can be by cryosurgery, 
laser surgery, or by the use of topical bleomycin. However, regardless of the extent of the lesion or of the modality of treatment, in as many as $30 \%$ of treated cases, leukoplakias will recur and treatment will not prevent the progression of some leukoplakias to squamous cell carcinoma $[5,15,21$, 32].

Idiopathic leukoplakia, non-homogeneous leukoplakia, leukoplakia affecting high-risk oral sites, and leukoplakia showing moderate or severe grades of epithelial dysplasia and particularly leukoplakias, in which a combination of these factors affect the risk of carcinomatous transformation, should be treated aggressively. Any changes in colour, texture or size, and appearance of additional leukoplakias at new oral sites are advance warning of the possibility of carcinomatous transformation.

\section{Summary}

Leukoplakia is the most common potentially malignant lesion of the mouth. It may unpredictably regress, may remain stable, or may undergo carcinomatous transformation. Those leukoplakias that are committed to a cancerous pathway most probably arise within a field of precancerized epithelium consisting of keratinocytes at different stages of cytogenetic transformation. This may explain the high rate of recurrence of oral leukoplakia despite treatment, and why some leukoplakias progress to carcinoma.

Many studies have reported the presence of HPV DNA in oral leukoplakias. However, there is not enough evidence to prove any casual association either between HPV and the development of oral leukoplakia, or between HPV and the progression of oral leukoplakia to carcinoma. The nature of the link between HPV infection and oral leukoplakia is as yet unknown.

\section{References}

[1] L. Feller and J. Lemmer, "Field cancerization and oral leukoplakia," in Field Cancerization: Basic Science and Clinical Applications, G. D. Dakubo, Ed., pp. 95-111, Nova Science, Ontario, Canada, 2011.

[2] J. J. Sciubba, "Oral cancer: the importance of early diagnosis and treatment," American Journal of Clinical Dermatology, vol. 2, no. 4, pp. 239-251, 2001.

[3] A. Roosaar, L. Yin, A. L. V. Johansson, G. Sandborgh-Englund, O. Nyrén, and T. Axéll, "A long-term follow-up study on the natural course of oral leukoplakia in a Swedish populationbased sample," Journal of Oral Pathology and Medicine, vol. 36, no. 2, pp. 78-82, 2007.

[4] T. Amagasa, M. Yamashiro, and N. Uzawa, "Oral premalignant lesions: from a clinical perspective," International Journal of Clinical Oncology, vol. 16, no. 1, pp. 5-14, 2011.

[5] I. van der Waal, "Potentially malignant disorders of the oral and oropharyngeal mucosa; terminology, classification and present concepts of management," Oral Oncology, vol. 45, no. 4-5, pp. 317-323, 2009.

[6] I. van der Waal, "Potentially malignant disorders of the oral and oropharyngeal mucosa; present concepts of management," Oral Oncology, vol. 46, no. 6, pp. 423-425, 2010.
[7] S. Warnakulasuriya, N. W. Johnson, and I. van der Waal, "Nomenclature and classification of potentially malignant disorders of the oral mucosa," Journal of Oral Pathology and Medicine, vol. 36, no. 10, pp. 575-580, 2007.

[8] B. Neville, D. Damm, C. Allen, and J. Bouquot, Oral and Maxillofacial Pathology, Saunders/Elsevier, St. Louis, Mo, USA, 3rd edition, 2009.

[9] T. Dietrich, P. A. Reichart, and C. Scheifele, "Clinical risk factors of oral leukoplakia in a representative sample of the US population," Oral Oncology, vol. 40, no. 2, pp. 158-163, 2004.

[10] G. Lodi, A. Sardella, C. Bez, F. Demarosi, and A. Carrassi, "Interventions for treating oral leukoplakia," Cochrane Database of Systematic Reviews, no. 4, Article ID CD001829, 2006.

[11] J. Reibel, "Prognosis of oral pre-malignant lesions: significance of clinical, histopathological, and molecular biological characteristics," Critical Reviews in Oral Biology and Medicine, vol. 14, no. 1, pp. 47-62, 2003.

[12] I. van der Waal and T. Axéll, "Oral leukoplakia: a proposal for uniform reporting," Oral Oncology, vol. 38, no. 6, pp. 521-526, 2002.

[13] S. S. Napier and P. M. Speight, "Natural history of potentially malignant oral lesions and conditions: an overview of the literature," Journal of Oral Pathology and Medicine, vol. 37, no. 1, pp. 1-10, 2008.

[14] C. Scully, J. Sudbø, and P. M. Speight, "Progress in determining the malignant potential of oral lesions," Journal of Oral Pathology and Medicine, vol. 32, no. 5, pp. 251-256, 2003.

[15] P. Holmstrup, P. Vedtofte, J. Reibel, and K. Stoltze, "Longterm treatment outcome of oral premalignant lesions," Oral Oncology, vol. 42, no. 5, pp. 461-474, 2006.

[16] P. A. Reichart and H. P. Philipsen, "Oral erythroplakia-a review," Oral Oncology, vol. 41, no. 6, pp. 551-561, 2005.

[17] L. S. Hansen, J. A. Olson, and S. Silverman Jr., "Proliferative verrucous leukoplakia. A long-term study of thirty patients," Oral Surgery, Oral Medicine, Oral Pathology, vol. 60, no. 3, pp. 285-298, 1985.

[18] J. M. Zakrzewska, V. Lopes, P. Speight, and C. Hopper, "Proliferative verrucous leukoplakia a report of ten cases," Oral Surgery, Oral Medicine, Oral Pathology, Oral Radiology, and Endodontology, vol. 82, no. 4, pp. 396-401, 1996.

[19] S. Silverman Jr. and M. Gorsky, "Proliferative verrucous leukoplakia: a follow-up study of 54 cases," Oral Surgery, Oral Medicine, Oral Pathology, Oral Radiology and Endodontology, vol. 84, no. 2, pp. 154-157, 1997.

[20] D. Greenspan and R. C. K. Jordan, "The white lesion that kills-aneuploid dysplastic oral leukoplakia," The New England Journal of Medicine, vol. 350, no. 14, pp. 1382-1384, 2004.

[21] J. V. Bagan, Y. Jimenez, J. M. Sanchis et al., "Proliferative verrucous leukoplakia: high incidence of gingival squamous cell carcinoma," Journal of Oral Pathology and Medicine, vol. 32, no. 7, pp. 379-382, 2003.

[22] L. Feller, N. H. Wood, and E. J. Raubenheimer, "Proliferative verrucous leukoplakia and field cancerization: report of a case," Journal of the International Academy of Periodontology, vol. 8, no. 2, pp. 67-70, 2006.

[23] R. J. Cabay, T. H. Morton Jr., and J. B. Epstein, "Proliferative verrucous leukoplakia and its progression to oral carcinoma: a review of the literature," Journal of Oral Pathology and Medicine, vol. 36, no. 5, pp. 255-261, 2007.

[24] J. V. Bagán, J. Murillo, R. Poveda, C. Gavaldá, Y. Jiménez, and C. Scully, "Proliferative verrucous leukoplakia: unusual 
locations of oral squamous cell carcinomas, and field cancerization as shown by the appearance of multiple OSCCs," Oral Oncology, vol. 40, no. 4, pp. 440-443, 2004.

[25] S. Petti, "Pooled estimate of world leukoplakia prevalence: a systematic review," Oral Oncology, vol. 39, no. 8, pp. 770-780, 2003.

[26] P. Suarez, J. G. Batsakis, and A. K. El-Naggar, "Leukoplakia: still a gallimaufry or is progress being made?-a review," Advances in Anatomic Pathology, vol. 5, no. 3, pp. 137-155, 1998.

[27] L. Feller, M. Altini, and H. Slabbert, "Pre-malignant lesions of the oral mucosa in a South African sample-a clinicopathological study," The Journal of the Dental Association of South Africa, vol. 46, no. 5, pp. 261-265, 1991.

[28] S. Warnakulasuriya, J. Reibel, J. Bouquot, and E. Dabelsteen, "Oral epithelial dysplasia classification systems: predictive value, utility, weaknesses and scope for improvement," Journal of Oral Pathology and Medicine, vol. 37, no. 3, pp. 127-133, 2008.

[29] S. Warnakulasuriya, "Histological grading of oral epithelial dysplasia: revisited," Journal of Pathology, vol. 194, no. 3, pp. 294-297, 2001.

[30] S. Silverman Jr., M. Gorsky, and F. Lozada, "Oral leukoplakia and malignant transformation. A follow-up study of 257 patients," Cancer, vol. 53, no. 3, pp. 563-568, 1984.

[31] S. Silverman Jr., M. Gorsky, and G. E. Kaugars, "Leukoplakia, dysplasia, and malignant transformation," Oral Surgery, Oral Medicine, Oral Pathology, Oral Radiology, and Endodontology, vol. 82 , no. 2 , article $117,1996$.

[32] G. Lodi and S. Porter, "Management of potentially malignant disorders: evidence and critique," Journal of Oral Pathology and Medicine, vol. 37, no. 2, pp. 63-69, 2008.

[33] M. Partridge, S. Pateromichelakis, E. Phillips, G. G. Emilion, R. P. A'Hern, and J. D. Langdon, "A case-control study confirms that microsatellite assay can identify patients at risk of developing oral squamous cell carcinoma within a field of cancerization," Cancer Research, vol. 60, no. 14, pp. 3893-3898, 2000.

[34] H. Lumerman, P. Freedman, and S. Kerpel, "Oral epithelial dysplasia and the development of invasive squamous cell carcinoma," Oral Surgery, Oral Medicine, Oral Pathology, Oral Radiology, and Endodontology, vol. 79, no. 3, pp. 321-329, 1995.

[35] M. P. Tabor, B. J. M. Braakhuis, J. E. van der Wal et al., "Comparative molecular and histological grading of epithelial dysplasia of the oral cavity and the oropharynx," Journal of Pathology, vol. 199, no. 3, pp. 354-360, 2003.

[36] P. Holmstrup, P. Vedtofte, J. Reibel, and K. Stoltze, "Oral premalignant lesions: is a biopsy reliable?" Journal of Oral Pathology and Medicine, vol. 36, no. 5, pp. 262-266, 2007.

[37] M. Weijers, I. Ten Hove, R. H. B. Allard, D. P. D. Bezemer, and I. van der Waal, "Patients with oral cancer developing from pre-existing oral leukoplakia: do they do better than those with de novo oral cancer?" Journal of Oral Pathology and Medicine, vol. 37, no. 3, pp. 134-136, 2008.

[38] L. Zhang, K. J. Cheung, W. L. Lam et al., "Increased genetic damage in oral leukoplakia from high risk sites: potential impact on staging and clinical management," Cancer, vol. 91, no. 11, pp. 2148-2155, 2001.

[39] S. Warnakulasuriya, "Lack of molecular markers to predict malignant potential of oral precancer," Journal of Pathology, vol. 190, no. 4, pp. 407-409, 2000.
[40] J. Sudbø, W. Kildal, B. Risberg, H. S. Koppang, H. E. Danielsen, and A. Reith, "DNA content as a prognostic marker in patients with oral leukoplakia," The New England Journal of Medicine, vol. 344, no. 17, pp. 1270-1278, 2001.

[41] M. P. Rosin, X. Cheng, C. Poh et al., "Use of allelic loss to predict malignant risk for low-grade oral epithelial dysplasia," Clinical Cancer Research, vol. 6, no. 2, pp. 357-362, 2000.

[42] L. Mao, J. S. Lee, Y. H. Fan et al., "Frequent microsatellite alterations at chromosomes 9p21 and 3p14 in oral premalignant lesions and their value in cancer risk assessment," Nature Medicine, vol. 2, no. 6, pp. 682-685, 1996.

[43] M. P. Tabor, R. H. Brakenhoff, V. M. M. van Houten et al., "Persistence of genetically altered fields in head and neck cancer patients: biological and clinical implications," Clinical Cancer Research, vol. 7, no. 6, pp. 1523-1532, 2001.

[44] J. Califano, P. van der Riet, W. Westra et al., "Genetic progression model for head and neck cancer: implications for field cancerization," Cancer Research, vol. 56, no. 11, pp. 24882492, 1996.

[45] J. J. Lee, W. K. Hong, W. N. Hittelman et al., "Predicting cancer development in oral leukoplakia: ten years of translational research," Clinical Cancer Research, vol. 6, no. 5, pp. 17021710, 2000.

[46] L. Zhang and M. P. Rosin, "Loss of heterozygosity: a potential tool in management of oral premalignant lesions?" Journal of Oral Pathology and Medicine, vol. 30, no. 9, pp. 513-520, 2001.

[47] M. M. Kim and J. A. Califano, "Molecular pathology of headand-neck cancer," International Journal of Cancer, vol. 112, no. 4, pp. 545-553, 2004.

[48] M. J. Conway and C. Meyers, "Replication and assembly of human papillomaviruses," Journal of Dental Research, vol. 88, no. 4, pp. 307-317, 2009.

[49] L. Feller, R. A. Khammissa, N. H. Wood, and J. Lemmer, "Epithelial maturation and molecular biology of oral HPV," Infectious Agents and Cancer, vol. 4, no. 1, article 16, 2009.

[50] L. Vidal and M. L. Gillison, "Human papillomavirus in HNSCC: recognition of a distinct disease type," Hematology/Oncology Clinics of North America, vol. 22, no. 6, pp. 11251142, 2008.

[51] L. Feller, N. H. Wood, R. A. G. Khammissa, and J. Lemmer, "Human papillomavirus-mediated carcinogenesis and HPVassociated oral and oropharyngeal squamous cell carcinomapart 2: human papillomavirus associated oral and oropharyngeal squamous cell carcinoma," Head \& Face Medicine, vol. 6, article 15, 2010.

[52] L. Feller, N. H. Wood, R. A. G. Khammissa, and J. Lemmer, "Human papillomavirus-mediated carcinogenesis and HPVassociated oral and oropharyngeal squamous cell carcinomapart 1: human papillomavirus-mediated carcinogenesis," Head \& Face Medicine, vol. 6, article 14, 2010.

[53] V. E. Furrer, M. B. Benitez, M. Furnes, H. E. Lanfranchi, and N. M. Modesti, "Biopsy vs. superficial scraping: detection of human papillomavirus $6,11,16$, and 18 in potentially malignant and malignant oral lesions," Journal of Oral Pathology and Medicine, vol. 35, no. 6, pp. 338-344, 2006.

[54] H. Nielsen, B. Norrild, P. Vedtofte, F. Prætorius, J. Reibel, and P. Holmstrup, "Human papillomavirus in oral premalignant lesions," European Journal of Cancer B, vol. 32, no. 4, pp. 264270, 1996.

[55] C. Ostwald, K. Rutsatz, J. Schweder, W. Schmidt, K. Gundlach, and M. Barten, "Human papillomavirus 6/11, 16 and 18 in oral carcinomas and benign oral lesions," Medical Microbiology and Immunology, vol. 192, no. 3, pp. 145-148, 2003. 
[56] F. Praetorius, "HPV-Associated diseases of oral mucosa," Clinics in Dermatology, vol. 15, no. 3, pp. 399-413, 1997.

[57] P. T. Hennessey, W. H. Westra, and J. A. Califano, "Human papillomavirus and head and neck squamous cell carcinoma: recent evidence and clinical implications," Journal of Dental Research, vol. 88, no. 4, pp. 300-306, 2009.

[58] P. K. Ha and J. A. Califano, "The role of human papillomavirus in oral carcinogenesis," Critical Reviews in Oral Biology and Medicine, vol. 15, no. 4, pp. 188-196, 2004.

[59] G. Campisi and L. Giovannelli, "Controversies surrounding human papilloma virus infection, head \& neck vs oral cancer, implications for prophylaxis and treatment," Head \& Neck Oncology, vol. 1, p. 8, 2009.

[60] C. G. L. Hobbs, J. A. C. Sterne, M. Bailey, R. S. Heyderman, M. A. Birchall, and S. J. Thomas, "Human papillomavirus and head and neck cancer: a systematic review and meta-analysis," Clinical Otolaryngology, vol. 31, no. 4, pp. 259-266, 2006.

[61] N. Termine, V. Panzarella, S. Falaschini et al., "HPV in oral squamous cell carcinoma vs head and neck squamous cell carcinoma biopsies: a meta-analysis (1988-2007)," Annals of Oncology, vol. 19, no. 10, pp. 1681-1690, 2008.

[62] A. R. Kreimer, G. M. Clifford, P. Boyle, and S. Franceschi, "Human papillomavirus types in head and neck squamous cell carcinomas worldwide: a systemic review," Cancer Epidemiology Biomarkers and Prevention, vol. 14, no. 2, pp. 467-475, 2005.

[63] C. S. Miller and B. M. Johnstone, "Human papillomavirus as a risk factor for oral squamous cell carcinoma: a meta-analysis, 1982-1997," Oral Surgery, Oral Medicine, Oral Pathology, Oral Radiology, and Endodontology, vol. 91, no. 6, pp. 622-635, 2001.

[64] L. Feller, N. H. Wood, R. A. G. Khammissa et al., "Epidemiology and transmission of HPV infection: vaccination for HPV infection," South African Dental Journal, vol. 65, pp. 478-481, 2010.

[65] J. M. Palefsky, S. Silverman Jr., M. Abdel-Salaam, T. E. Daniels, and J. S. Greenspan, "Association between proliferative verrucous leukoplakia and infection with human papillomavirus type 16," Journal of Oral Pathology and Medicine, vol. 24, no. 5, pp. 193-197, 1995.

[66] M. Bouda, V. G. Gorgoulis, N. G. Kastrinakis et al., "“High risk" HPV types are frequently detected in potentially malignant and malignant oral lesions, but not in normal oral mucosa," Modern Pathology, vol. 13, no. 6, pp. 644-653, 2000.

[67] J. Pintos, M. J. Black, N. Sadeghi et al., "Human papillomavirus infection and oral cancer: a case-control study in Montreal, Canada," Oral Oncology, vol. 44, no. 3, pp. 242-250, 2008.

[68] L. Charfi, T. Jouffroy, P. de Cremoux et al., "Two types of squamous cell carcinoma of the palatine tonsil characterized by distinct etiology, molecular features and outcome," Cancer Letters, vol. 260, no. 1-2, pp. 72-78, 2008.

[69] P. M. Weinberger, Z. Yu, B. G. Haffty et al., "Molecular classification identifies a subset of human papillomavirusassociated oropharyngeal cancers with favorable prognosis," Journal of Clinical Oncology, vol. 24, no. 5, pp. 736-747, 2006.

[70] L. Licitra, F. Perrone, P. Bossi et al., "High-risk human papillomavirus affects prognosis in patients with surgically treated oropharyngeal squamous cell carcinoma," Journal of Clinical Oncology, vol. 24, no. 36, pp. 5630-5636, 2006.

[71] B. J. M. Braakhuis, P. J. F. Snijders, W. J. H. Keune et al., "Genetic patterns in head and neck cancers that contain or lack transcriptionally active human papillomavirus," Journal of the National Cancer Institute, vol. 96, no. 13, pp. 998-1006, 2004.

[72] W. J. Koskinen, R. W. Chen, I. Leivo et al., "Prevalence and physical status of human papillomavirus in squamous cell carcinomas of the head and neck," International Journal of Cancer, vol. 107, no. 3, pp. 401-406, 2003.

[73] J. P. Klussmann, S. J. Weissenborn, U. Wieland et al., "Prevalence, distribution, and viral load of human papillomavirus 16 DNA in tonsillar carcinomas," Cancer, vol. 92, no. 11, pp. 2875-2884, 2001.

[74] T. Wiest, E. Schwarz, C. Enders, C. Flechtenmacher, and F. X. Bosch, "Involvement of intact HPV16 E6/E7 gene expression in head and neck cancers with unaltered p53 status and perturbed pRB cell cycle control," Oncogene, vol. 21, no. 10, pp. 1510-1517, 2002.

[75] X. H. Liang, J. Lewis, R. Foote, D. Smith, and D. Kademani, "Prevalence and significance of human papillomavirus in oral tongue cancer: the Mayo Clinic experience," Journal of Oral and Maxillofacial Surgery, vol. 66, no. 9, pp. 1875-1880, 2008.

[76] P. K. Ha, S. I. Pai, W. H. Westra et al., "Real-time quantitative PCR demonstrates low prevalence of human papillomavirus type 16 in premalignant and malignant lesions of the oral cavity," Clinical Cancer Research, vol. 8, no. 5, pp. 1203-1209, 2002.

[77] V. M. M. van Houten, P. J. F. Snijders, M. W. M. van den Brekel et al., "Biological evidence that human papillomaviruses are etiologically involved in a subgroup of head and neck squamous cell carcinomas," International Journal of Cancer, vol. 93, no. 2, pp. 232-235, 2001.

[78] G. Campisi, L. Giovannelli, P. Aricò et al., "HPV DNA in clinically different variants of oral leukoplakia and lichen planus," Oral Surgery, Oral Medicine, Oral Pathology, Oral Radiology and Endodontology, vol. 98, no. 6, pp. 705-711, 2004. 


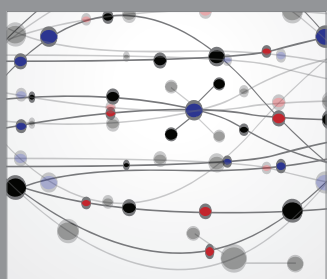

The Scientific World Journal
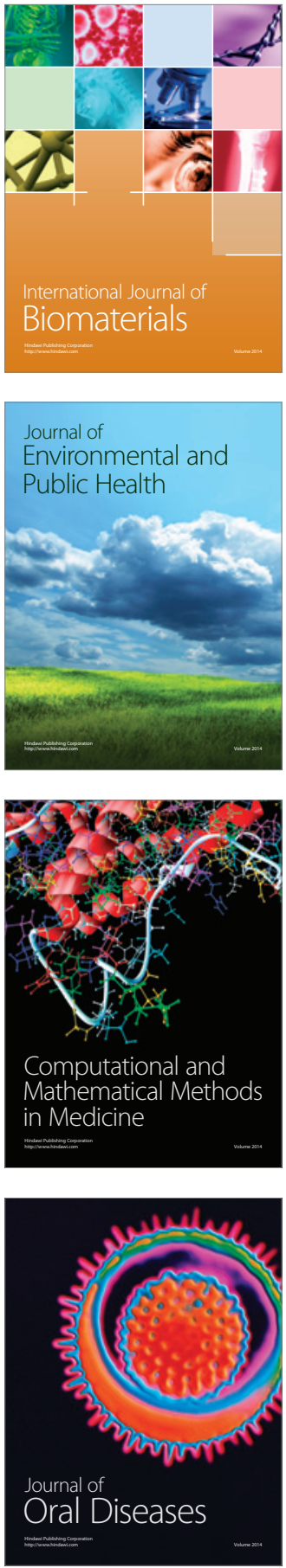
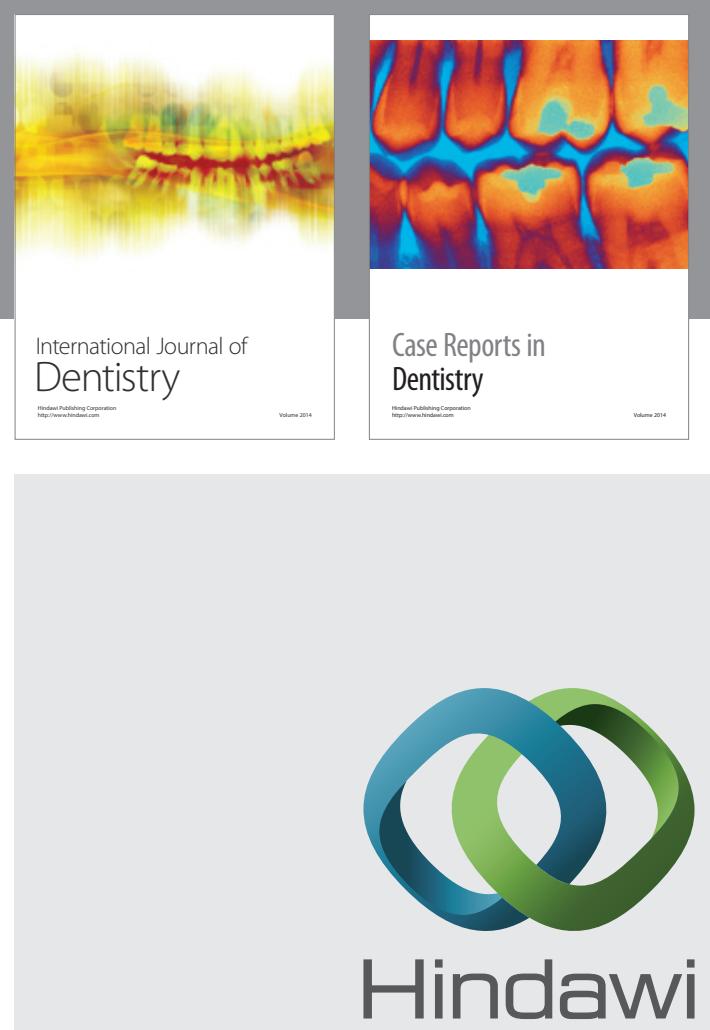

Submit your manuscripts at

http://www.hindawi.com
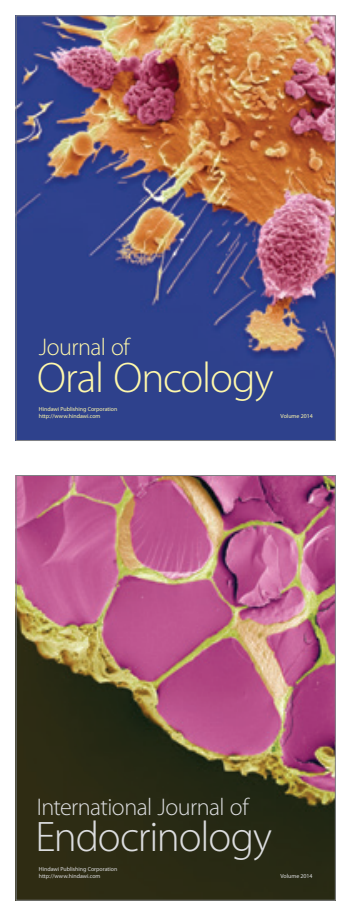
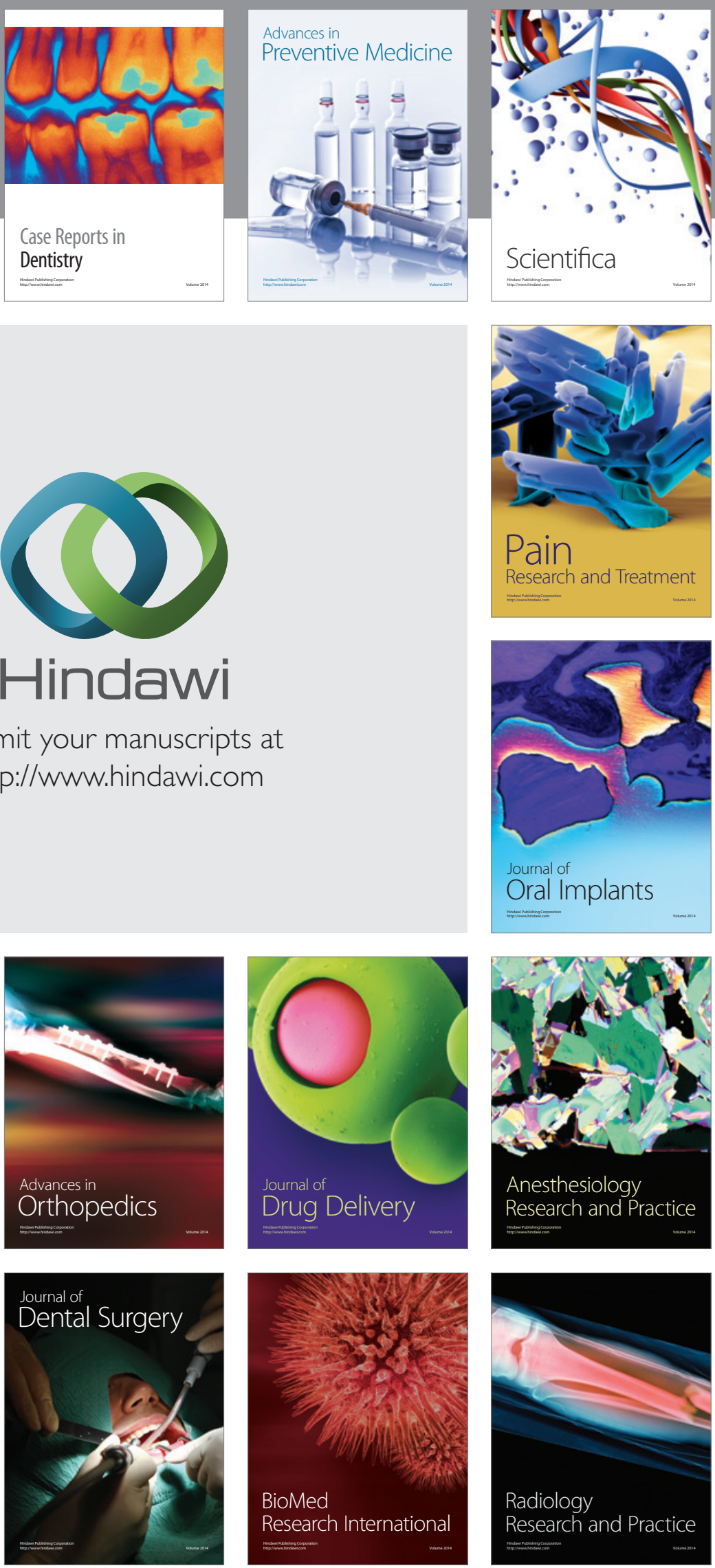